Supplement of Biogeosciences, 16, 2329-2341, 2019

https://doi.org/10.5194/bg-16-2329-2019-supplement

(C) Author(s) 2019. This work is distributed under

the Creative Commons Attribution 4.0 License.

(c) (1)

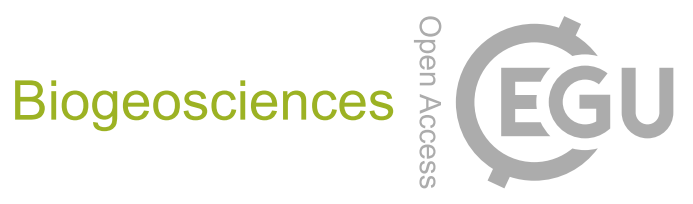

Supplement of

\title{
Responses of an abyssal meiobenthic community to short-term burial with crushed nodule particles in the south-east Pacific
}

Lisa Mevenkamp et al.

Correspondence to: Lisa Mevenkamp (lisa.mevenkamp@ugent.be)

The copyright of individual parts of the supplement might differ from the CC BY 4.0 License. 

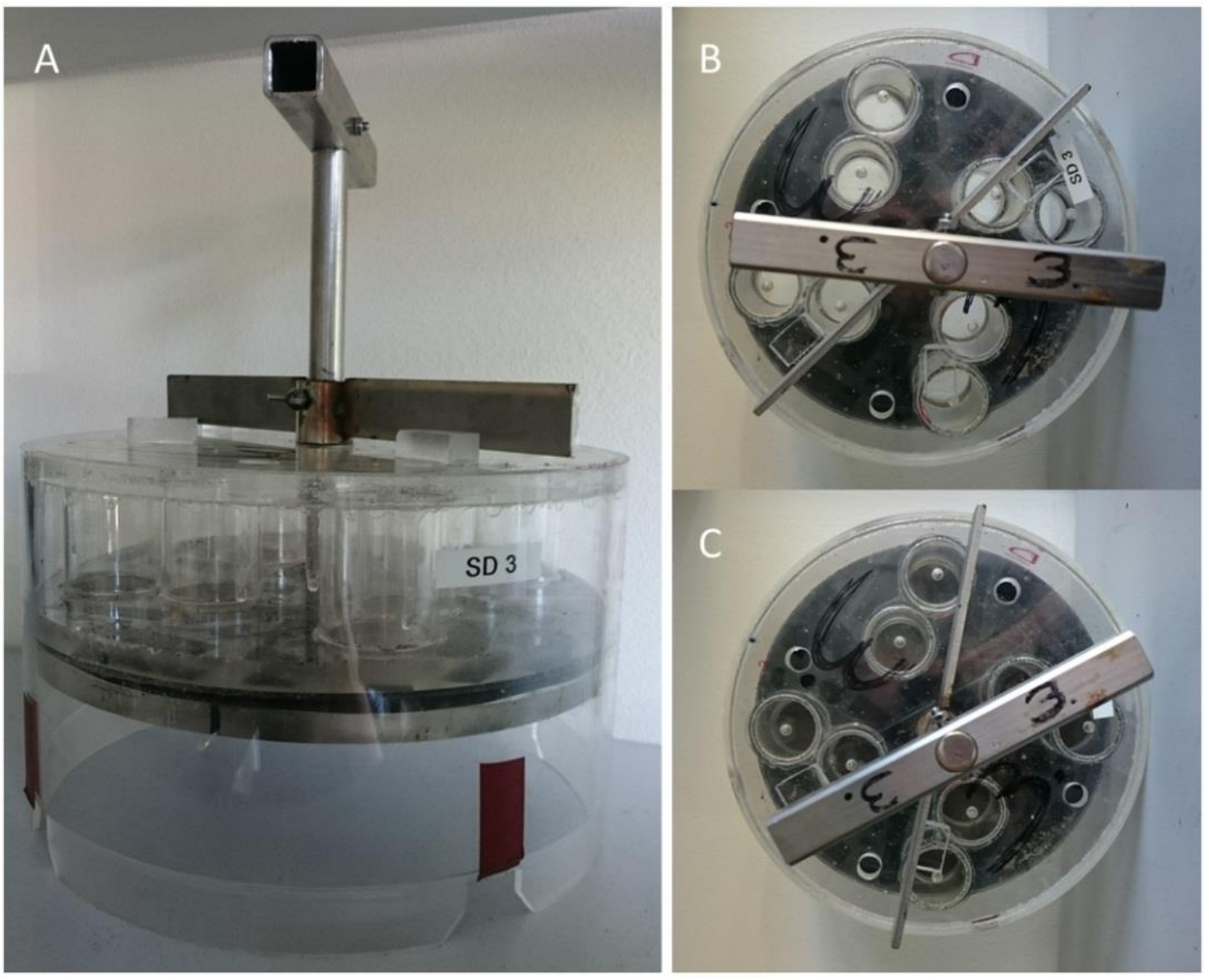

Figure S1 A) Front view on the sediment-dispensing device, sediment is filled in tubes inside the round plexiglass space B) Top view 10 in open position, tubes are visible as big holes and C) Top view in closed position. Holes in the plexiglass cover ensured escape of all air in the device. 


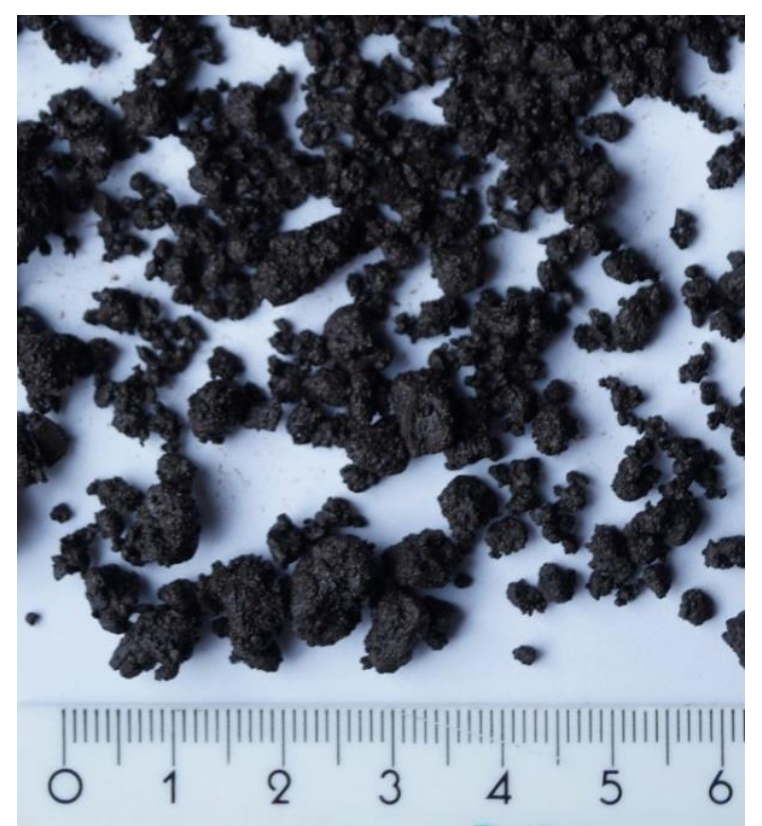

Figure S2 Example of the crushed nodule substrate. Scale in centimetres.
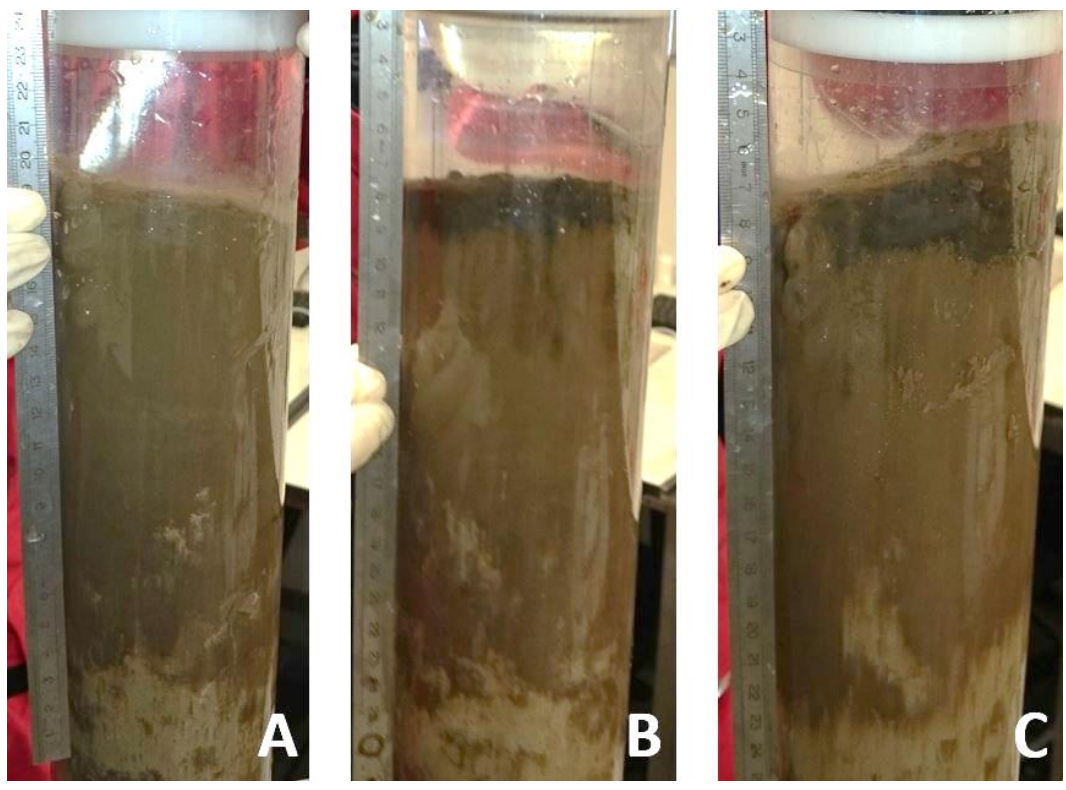

5 Figure S3 Pictures of push cores taken at the end of the experiment from the Control treatment (A) and the Burial treatment (B and C). Through its black colour, the layer of crushed nodule debris is easily distinguishable from the underlying sediment. 


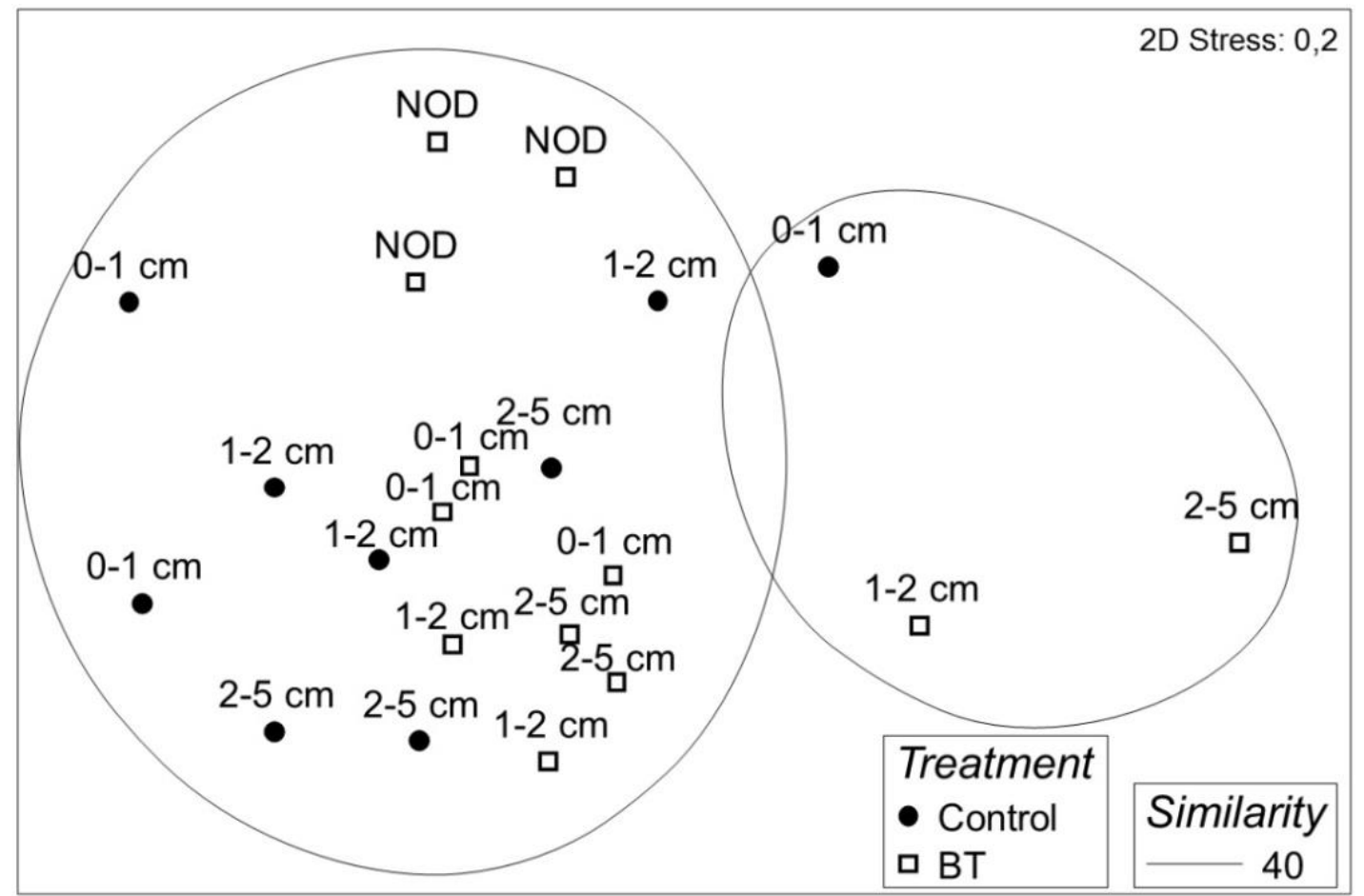

Figure S4 MDS plot of the relative nematode genus composition in each sample of the Control and Burial treatment (BT) per sediment depth layer with overlying contours of significant (SIMPROF test) clusters at a $40 \%$ similarity level. NOD = crushed nodule layer 
Table S1 Mean densities (ind. $10 \mathrm{~cm}^{-2}, \pm$ standard error)and feeding type group of nematode genera found in both treatments of the experiment combining all depth layers.

\begin{tabular}{|c|c|c|c|c|c|c|c|}
\hline \multirow{2}{*}{$\begin{array}{l}\text { Order } \\
\text { Araeolaimida }\end{array}$} & \multirow{2}{*}{$\begin{array}{l}\text { Family } \\
\text { Axonolaimidae }\end{array}$} & \multirow{2}{*}{$\frac{\text { Genus }}{\text { Ascolaimus }}$} & \multirow{2}{*}{$\begin{array}{c}\begin{array}{c}\text { Feeding } \\
\text { type }\end{array} \\
1 \mathrm{~B}\end{array}$} & \multicolumn{2}{|c|}{ Control } & \multicolumn{2}{|c|}{ Burial treatment } \\
\hline & & & & & & 0.17 & \pm 0.17 \\
\hline & Comesomatidae & Cervonema & $1 \mathrm{~B}$ & 3.57 & \pm 0.81 & 2.59 & \pm 0.90 \\
\hline & & Minolaimus & $1 \mathrm{~A}$ & 0.43 & \pm 0.43 & 0.29 & \pm 0.29 \\
\hline & & Pierrickia & $1 \mathrm{~B}$ & 0.42 & \pm 0.21 & 0.94 & \pm 0.04 \\
\hline & Coninckiidae & Coninckia & $1 \mathrm{~A}$ & & & 0.15 & \pm 0.15 \\
\hline & Diplopeltidae & Campylaimus & $1 \mathrm{~B}$ & 0.21 & \pm 0.21 & 0.15 & \pm 0.15 \\
\hline & & Diplopeltula & $1 \mathrm{~A}$ & 2.29 & \pm 0.73 & 1.72 & \pm 0.17 \\
\hline & & Intasia & $1 \mathrm{~A}$ & 0.62 & \pm 0.35 & & \\
\hline \multirow[t]{17}{*}{ Chromadorida } & Chromadoridae & Acantholaimus & $2 \mathrm{~A}$ & 12.49 & \pm 1.13 & 16.12 & \pm 2.06 \\
\hline & & Actinonema & $2 \mathrm{~A}$ & 1.04 & \pm 0.41 & 0.78 & \pm 0.57 \\
\hline & & Chromadora & $2 A$ & 0.62 & \pm 0.62 & 0.30 & \pm 0.15 \\
\hline & & Chromadorina & $2 \mathrm{~A}$ & 0.62 & \pm 0.36 & 0.48 & \pm 0.27 \\
\hline & & Endeolophos & $2 A$ & & & 0.47 & \pm 0.02 \\
\hline & & Hypodontolaimus & $2 A$ & & & 0.16 & \pm 0.16 \\
\hline & & Prochromadora & $2 A$ & & & 0.15 & \pm 0.15 \\
\hline & & Prochromadorella & $2 \mathrm{~A}$ & 0.41 & \pm 0.41 & 0.47 & \pm 0.47 \\
\hline & & Spilophorella & $2 \mathrm{~A}$ & 0.42 & \pm 0.21 & 0.17 & \pm 0.17 \\
\hline & Cyatholaimidae & Acantonchus & $2 \mathrm{~A}$ & 0.43 & \pm 0.43 & & \\
\hline & & Longicyatholaimus & $2 A$ & 0.20 & \pm 0.20 & & \\
\hline & & Marylynnia & $2 A$ & & & 0.15 & \pm 0.15 \\
\hline & & Paracantonchus & $2 A$ & & & 0.15 & \pm 0.15 \\
\hline & & Paracyatholaimus & $2 \mathrm{~A}$ & & & 0.17 & \pm 0.17 \\
\hline & & Pomponema & $2 \mathrm{~B}$ & 0.21 & \pm 0.21 & 0.15 & \pm 0.15 \\
\hline & Neotonchidae & Gomphionchus & & & & 0.17 & \pm 0.17 \\
\hline & Selachinematidae & Synonchiella & $2 \mathrm{~B}$ & 0.22 & \pm 0.22 & 0.17 & \pm 0.17 \\
\hline \multirow[t]{7}{*}{ Desmodorida } & Desmodoridae & Desmodora & $2 \mathrm{~A}$ & 1.70 & \pm 1.14 & 1.90 & \pm 0.34 \\
\hline & & Desmodorella & $2 A$ & 0.20 & \pm 0.20 & & \\
\hline & & Metadesmodora & $1 \mathrm{~B}$ & 0.21 & \pm 0.21 & & \\
\hline & & Molgolaimus & $1 \mathrm{~A}$ & 0.85 & \pm 0.57 & 1.23 & \pm 0.40 \\
\hline & & Paradesmodora & $2 \mathrm{~A}$ & 0.21 & \pm 0.21 & & \\
\hline & Microlaimidae & Calomicrolaimus & $2 \mathrm{~A}$ & & & 0.31 & \pm 0.31 \\
\hline & & Microlaimus & $2 \mathrm{~A}$ & 1.05 & \pm 0.55 & 1.62 & \pm 0.75 \\
\hline \multirow[t]{6}{*}{ Desmoscolecida } & Cyartonematidae & Cyartonema & $1 \mathrm{~A}$ & 0.20 & \pm 0.20 & 0.49 & \pm 0.29 \\
\hline & & Southerniella & $1 \mathrm{~A}$ & 0.63 & \pm 0.36 & 0.46 & \pm 0.27 \\
\hline & Desmoscolecidae & Desmoscolex & $1 \mathrm{~A}$ & 3.31 & \pm 1.73 & 2.51 & \pm 1.06 \\
\hline & & Greeffiella & $1 \mathrm{~A}$ & 0.62 & \pm 0.35 & 0.96 & \pm 0.54 \\
\hline & & Hapalomus & $1 \mathrm{~A}$ & & & 0.31 & \pm 0.31 \\
\hline & & Tricoma & $1 \mathrm{~A}$ & 1.24 & \pm 0.35 & 1.89 & \pm 1.19 \\
\hline \multirow[t]{5}{*}{ Enoplida } & Anticomidae & Cephalanticoma & $2 \mathrm{~A}$ & & & 0.16 & \pm 0.16 \\
\hline & Enchelidiidae & Bathyeurystomina & $2 \mathrm{~B}$ & 0.42 & \pm 0.21 & 0.45 & \pm 0.25 \\
\hline & & Calyptronema & $1 b$ & & & 0.15 & \pm 0.15 \\
\hline & Enoplidae & Enoploides & $2 \mathrm{~B}$ & 0.22 & \pm 0.22 & & \\
\hline & & Mesacanthion & $2 \mathrm{~B}$ & & & 0.30 & \pm 0.15 \\
\hline
\end{tabular}




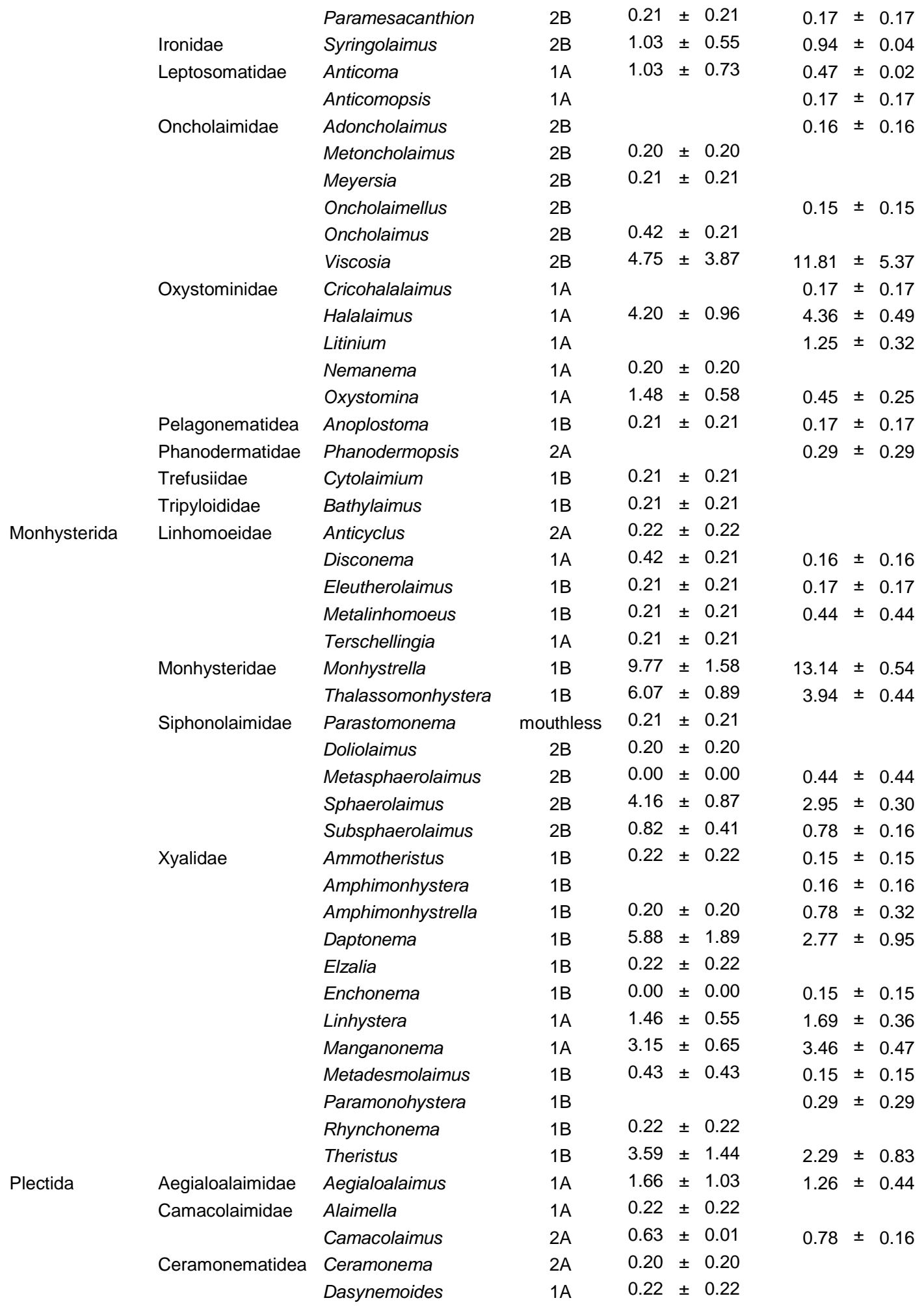


Pselionema

Diplopeltoididae

Haliplectidae

Leptolaimidae

Setoplectus

Antomicron
Diplopeltoides

Leptolaimus

Unknown sp. 1

Unknown sp. 2

Unknown sp. 3

Unknown sp. 4

Unknown sp. 5 $\begin{array}{lllll}1 \mathrm{~A} & 1.47 \pm 0.42 & 0.29 \pm 0.29\end{array}$

$1 \mathrm{~A} \quad 5.21 \pm 2.50 \quad 1.76 \pm 0.70$

$\begin{array}{lllll}1 \mathrm{~A} & 0.22 \pm 0.22 & 0.29 \pm 0.29\end{array}$

$1 \mathrm{~A} \quad 0.21 \pm 0.21 \quad 0.32 \pm 0.16$

$\begin{array}{llll}1 \mathrm{~A} & 2.49 \pm 0.70 & \pm .65 \pm 0.45\end{array}$

$0.22 \pm 0.22$

$0.63 \pm 0.16$

$0.47 \pm 0.47$

$0.17 \pm 0.17$

$0.15 \pm 0.15$ 
Table S2 Results of the SIMPER analysis between the significantly different clusters identified in the dataset of relative abundances of nematode feeding types. Av.Abund = average abundance, Av.Diss = average dissimilarity, Diss/SD = average contribution divided by the standard deviation, Contrib $\%=$ Contribution to the dissimilarities, Cum $\%=$ Cumulative contribution.

\begin{tabular}{lrrrrrr} 
Feeding type & $\begin{array}{c}\text { Cluster A } \\
\text { Av.Abund }\end{array}$ & Av.Abund & Av.Diss & Diss/SD & Contrib\% & Cum.\% \\
\hline 2B & 16 & 33 & 9 & 3 & 34 & 34 \\
2A & 37 & 21 & 8 & 2 & 30 & 65 \\
1A & 30 & 21 & 5 & 2 & 20 & 84 \\
1B & 17 & 25 & 4 & 2 & 16 & 100
\end{tabular}

Cluster A Cluster B

\begin{tabular}{lrrrrrr} 
Feeding type & Av.Abund & Av.Abund & Av.Diss & Diss/SD & Contrib\% & Cum.\% \\
\hline 1B & 17 & 36 & 9 & 2 & 37 & 37 \\
2A & 37 & 21 & 8 & 3 & 31 & 68 \\
1A & 30 & 34 & 4 & 1 & 16 & 84 \\
2B & 16 & 8 & 4 & 2 & 16 & 100
\end{tabular}

\begin{tabular}{lrrrrrr} 
Feeding type & $\begin{array}{l}\text { Cluster A } \\
\text { Av.Abund }\end{array}$ & Av.Abund & Av.Diss & Diss/SD & Contrib\% & Cum.\% \\
\hline 2B & 16 & 67 & 26 & 12 & 48 & 48 \\
2A & 37 & 9 & 14 & 8 & 27 & 75 \\
1A & 30 & 7 & 12 & 5 & 22 & 96
\end{tabular}

\begin{tabular}{lrrrrrr} 
& Cluster B & Cluster C & & & \\
Feeding type & Av.Abund & Av.Abund & Av.Diss & Diss/SD & Contrib\% & Cum.\% \\
\hline 2B & 8 & 33 & 13 & 4 & 43 & 43 \\
1A & 34 & 21 & 7 & 2 & 25 & 69 \\
1B & 36 & 25 & 6 & 2 & 20 & 89 \\
2A & 21 & 21 & 3 & 1 & 11 & 100
\end{tabular}

\begin{tabular}{lrrrrrr} 
& Cluster B & Cluster D & & & & \\
Feeding type & Av.Abund & Av.Abund & Av.Diss & Diss/SD & Contrib\% & Cum.\% \\
\hline 2B & 8 & 67 & 29 & 14 & 50 & 50 \\
1A & 34 & 7 & 14 & 3 & 23 & 73 \\
1B & 36 & 18 & 9 & 2 & 16 & 89 \\
2A & 21 & 9 & 6 & 3 & 11 & 100
\end{tabular}

\begin{tabular}{lrrrrrr}
\multicolumn{2}{c}{ Cluster C } & Cluster D & & & \\
Feeding type & Av.Abund & Av.Abund & Av.Diss & Diss/SD & Contrib\% & Cum.\% \\
\hline 2B & 33 & 67 & 17 & 5 & 50 & 50
\end{tabular}




$\begin{array}{rrrrrrr}1 \mathrm{~A} & 21 & 7 & 7 & 2 & 20 & 70 \\ 2 \mathrm{~A} & 21 & 9 & 6 & 2 & 19 & 89 \\ 1 \mathrm{~B} & 25 & 18 & 4 & 2 & 11 & 100\end{array}$

